\title{
On the generalized Hartley and Hartley-Hilbert transformations
}

\author{
Shrideh Khalaf Qasem Al-Omari ${ }^{1}$ and Adem Kılıçman²*
}

\author{
*Correspondence: \\ akilicman@putra.upm.edu.my \\ ${ }^{2}$ Department of Mathematics and \\ Institute of Mathematical Research, \\ Universiti Putra Malaysia (UPM), \\ Serdang, Selangor 43400, Malaysia \\ Full list of author information is \\ available at the end of the article
}

\begin{abstract}
In this paper, we extend Hartley and Hartley-Hilbert transformations (HT and HHT, respectively) to a certain space of tempered distributions. We then establish a certain convolution theorem for the HHT. The convolution theorem, obtained in this way, has been shown to possess a factorization property of Fourier convolution type. Proving the new convolution theorem for the HHT, by the usual convolution product, the transform is investigated on a certain space of Boehmians. Its properties of linearity and convergence are also discussed in the context of Boehmian spaces.
\end{abstract}

MSC: Primary 54C40; 14E20; secondary 46E25; $20 \mathrm{C} 20$

Keywords: distributions; test function; $\mathrm{HH}$ transform; Boehmians

\section{Test function spaces and distributions}

The idea of specifying a function not by its values but by its behavior as a functional on some space of testing functions is a concept that is quite familiar to scientists and engineers through their experience with the classical Fourier and Laplace transformations. Test functions, on which distributions operate, cannot in general be written down in an explicit form. The advantage of distributions over classical functions is that the distribution concept provides a better mechanism for analyzing certain physical phenomena than the function concept does because, for one reason, various entities such as the delta function $\delta$ can be correctly described as a distribution but not as a function. Furthermore, physical quantities that can be adequately represented as a function can also be characterized as a distribution. In addition, distributions attain an infinite number of derivatives and those derivatives always exist, which is not applied to functions.

The space of testing functions, denoted by $\mathcal{D}$, consists of all complex-valued functions $\varphi$ that are infinitely smooth and zero outside some finite interval. The set of continuous linear forms (conjugates or dual space) on $\mathcal{D}$ constitutes a space of distributions, denoted by $\mathcal{D}^{\prime}$.

A distribution $f$ in $\mathcal{D}^{\prime}$ can be represented, corresponding to $f(t)$, through the convergent integral

$$
\langle f, \varphi\rangle=\langle f(t), \varphi(t)\rangle \triangleq \int_{-\infty}^{\infty} f(t) \varphi(t) d t .
$$

The space of complex-valued smooth functions is denoted by $\mathcal{E}$ and its dual space is denoted by $\mathcal{E}^{\prime}$.

(c) 2013 Al-Omari and Kilıçman; licensee Springer. This is an Open Access article distributed under the terms of the Creative Commons Attribution License (http://creativecommons.org/licenses/by/2.0), which permits unrestricted use, distribution, and reproduction in any medium, provided the original work is properly cited. 
By $\mathcal{S}$ we denote the space of all complex-valued functions $\varphi$ that are infinitely smooth and are such that as $|t| \rightarrow \infty$, they and their partial derivatives decay to zero faster than all powers of $|t|^{-1}$. Elements of $\mathcal{S}$ are called testing functions of rapid descents. $\mathcal{S}$ is indeed a linear space.

If $\phi \in \mathcal{S}$, then its partial derivatives are in $\mathcal{S}$. In fact, $\mathcal{D}$ is dense in $\mathcal{S}$ and $\mathcal{S}$ is dense in $\mathcal{E}$. The dual space of $\mathcal{S}$ is called the space of tempered distributions and denoted by $\mathcal{S}^{\prime}$ with a property that $\mathcal{E}^{\prime} \subset \mathcal{S}^{\prime} \subset \mathcal{D}^{\prime}, \mathcal{E}^{\prime}$ being the (conjugate of $\mathcal{E}$ ) space of distributions of compact support. For the convergence on $\mathcal{D}, \mathcal{E}$ and $\mathcal{S}$ and their topologies, we refer to [1, 2].

\section{$2 \mathrm{HT}$ and HHT of tempered distributions}

\subsection{Introduction to HT and HH transforms}

The Hartley transform (HT) was introduced originally by Hartley 1942 as an integral transform with a number of properties similar to those of the Fourier transform (FT). The HT of a function over $\mathrm{R}$ is a real function defined by $[3,4]$

$$
(\mathrm{H} f)(x)=\int_{-\infty}^{\infty} f(y)(\cos (x y)+\sin (x y)) d y
$$

or

$$
(\mathrm{H} f)(x)=\int_{-\infty}^{\infty} f(y)(\cos (2 \pi x y)+\sin (2 \pi x y)) d y
$$

Some properties of HT are:

(i) Shift: $\left(\mathrm{H} f\left(y-y_{0}\right)\right)(x)=\cos \left(2 \pi x y y_{o}\right)(\mathrm{H} f)(x)+\sin \left(2 \pi x y_{o}\right)(\mathrm{H} f)(-x)$.

(ii) Modulation: $\mathrm{H}\left(\cos \left(2 \pi x_{o} y\right) f(y)\right)(x)=\frac{1}{2} \mathrm{H} f\left(x-x_{o}\right)+\frac{1}{2} \mathrm{H} f\left(x-x_{o}\right)$.

(iii) Derivative: $\mathrm{H}\left(\frac{d}{d y} f(y)\right)(x)=-2 \pi x(\mathrm{H} f)(-x)$.

(iv) Convolution: The convolution theorem of HT is given as

$$
\begin{aligned}
(\mathrm{H}(f * g)(t))(x)= & \frac{1}{2}((\mathrm{H} f)(x)(\mathrm{H} g)(x)+(\mathrm{H} f)(-x)(\mathrm{H} g)(x)) \\
& +\frac{1}{2}((\mathrm{H} f)(x)(\mathrm{H} g)(-x)-(\mathrm{H} f)(-x)(\mathrm{H} g)(x)) .
\end{aligned}
$$

The Hilbert transform via the Hartley transform, the Hartley-Hilbert transform (HHT), is defined by [5]

$$
(\mathrm{HH} f)(x) \triangleq \frac{1}{\pi} \int_{0}^{\infty}\left(\mathrm{H}^{o} f(y) \cos (x y)+\mathrm{H}^{e} f(y) \sin (x y)\right) d y,
$$

where

$$
\mathrm{H}^{o} f(y)=\frac{\mathrm{H} f(y)-\mathrm{H} f(-y)}{2}, \quad \mathrm{H}^{e} f(y)=\frac{\mathrm{H} f(y)+\mathrm{H} f(-y)}{2}
$$

are the odd and even components of the HT.

The HHT, which permits some attractive applications in geophysics and signal processing, has been extended to a specific space of generalized functions (Boehmian spaces) in [6]. 


\subsection{First convolution theorem of HHT}

Convolutions of integral transforms which possess the factorization property of Fourier convolution type have become of interest to many authors and have been applied to solving systems of integral equations. In [7], we studied the convolution theorem for HHT in some detail. In this paper we make the idea more precise. We define some generalized convolution of HHT that permits a factorization property of Fourier convolution type.

Let $U$ be a linear space and $V$ be a commutative algebra on the field $K$. Let $T \in \mathrm{L}(\mathrm{U}, \cdot)$ be a linear operator from $\mathrm{U}$ to $\mathrm{V}$. A bilinear map $\star: \mathrm{U} \times \mathrm{U}: \rightarrow \mathrm{U}$ is called the convolution for $\mathrm{T}$ if $\mathrm{T}(\star(f, g))=\mathrm{T}(f) \mathrm{T}(g)$ for any $f, g \in \mathrm{U}$. The image $\star(f, g)$ is denoted by $f \star g$.

Theorem 2.1 (First convolution theorem of HHT) Let $\mathrm{HH} f, \mathrm{HH} g$ be the HHTs of $f$ and $g$, respectively, then

$$
\mathrm{HH}(f \star g)(x)=(\mathrm{HH} f)(x)(\mathrm{HH} g)(x),
$$

where

$$
(f \star g)(t)=\int_{0}^{\infty}\left(f(t) \mathrm{H}^{o} g(\eta) \cos (x \eta)+f(t) \mathrm{H}^{e} g(\eta) \sin (x \eta)\right) d \eta .
$$

Proof Under the hypothesis of the theorem, we write

$$
\begin{aligned}
(\mathrm{HH} f)(x)(\mathrm{HH} g)(x) \\
=\int_{0}^{\infty}\left(\left(\mathrm{H}^{o} f\right)(\xi) \cos (x \xi)+\left(\mathrm{H}^{e} f\right)(\xi) \sin (x \xi)\right) d \xi \\
\quad \times \int_{0}^{\infty}\left(\left(\mathrm{H}^{o} g\right)(\eta) \cos (x \eta)+\left(\mathrm{H}^{e} g\right)(\eta) \sin (x \eta)\right) d \eta \\
=\int_{0}^{\infty}\left(\int_{0}^{\infty}\left(\mathrm{H}^{o} f(\xi) \mathrm{H}^{o} g(\eta) \cos (x \eta)+\mathrm{H}^{o} g(\xi) \mathrm{H}^{e} g(\eta) \sin (x \eta)\right) d \eta\right) \cos x \xi d \xi \\
\quad+\int_{0}^{\infty}\left(\int_{0}^{\infty}\left(\mathrm{H}^{e} f(\xi) \mathrm{H}^{o} g(\eta) \cos (x \eta)+\mathrm{H}^{o} f(\xi) \mathrm{H}^{e} g(\eta) \sin (x \eta)\right) d \eta\right) \sin x \xi d \xi,
\end{aligned}
$$

which can be written as

$$
(\mathrm{HH} f)(x)(\mathrm{HH} g)(x)=\int_{0}^{\infty}(\mathrm{A}(\xi) \cos (x \xi)+\mathrm{B}(\xi) \sin (x \xi)) d \xi
$$

where

$$
\mathrm{A}(\xi)=\int_{0}^{\infty}\left(\mathrm{H}^{o} f(\xi) \mathrm{H}^{o} g(\eta) \cos (x \eta)+\mathrm{H}^{o} f(\xi) \mathrm{H}^{e} g(\eta) \sin (x \eta)\right) d \eta
$$

and

$$
\mathrm{B}(\xi)=\int_{0}^{\infty}\left(\mathrm{H}^{e} f(\xi) \mathrm{H}^{o} g(\eta) \cos (x \eta)+\mathrm{H}^{o} f(\xi) \mathrm{H}^{e} g(\eta) \sin (x \eta)\right) d \eta .
$$


Then, with a simple modification, we get

$$
\begin{aligned}
\mathrm{A}(\xi) & =\int_{-\infty}^{\infty}\left(\int_{0}^{\infty}\left(f(t) \mathrm{H}^{o} g(\eta) \cos (x \eta)+f(t) \mathrm{H}^{e} g(\eta) \sin (x \eta)\right) d \eta\right) \sin (t \xi) d \xi \\
& =\int_{-\infty}^{\infty}(f \star g)(t) \sin (t \xi) d \xi,
\end{aligned}
$$

where

$$
(f \star g)(t)=\int_{0}^{\infty}\left(f(t) \mathrm{H}^{o} g(\eta) \cos (x \eta)+f(t) \mathrm{H}^{e} g(\eta) \sin (x \eta)\right) d \eta
$$

Similarly, we proceed for $\mathrm{B}(\xi)$ to get $\mathrm{B}(\xi)=\mathrm{H}^{e}(f \star g)(\xi), f \star g$ is the integral equation given in (7).

Hence the theorem.

Theorem 2.2 Let $f, g$ and $h$ be $L^{1}$ functions, then:

(i) $\mathrm{HH}(f \star g)=\mathrm{HH}(g \star f)$.

(ii) $\mathrm{HH}((f \star g) \star h)=\mathrm{HH}(f \star(g \star h))=\mathrm{HH}(g \star(f \star h))=\mathrm{HH}(h \star(f \star g))$.

(iii) $\mathrm{HH}(f \star(g+h))=\mathrm{HH}(f \star g)+\mathrm{HH}(f \star h)$.

(iv) $\mathrm{HH}(f+(g \star h))=\mathrm{HH}((f+g) \star(f \star h))$.

Proof (i) Let $f, g \in L^{1}$. By the aid of Theorem 2.1, we get

$$
\mathrm{HH}(f \star g)(x)=(\mathrm{HH} f)(x)(\mathrm{HH} g)(x)=(\mathrm{HH} g)(x)(\mathrm{HH} f)(x)=\mathrm{HH}(g \star f)(x) .
$$

Proof of (ii) is analogous to that of the first part.

(iii) Let $f, g \in L^{1}$, then using the definition of HHT and that of $\star$, we get

$$
\begin{aligned}
& \mathrm{HH}(f \star(g+h))(x) \\
& \quad=\frac{1}{\pi} \int_{0}^{\infty}\left(\mathrm{H}^{o}(f \star(g+h))(y) \cos (x y)+\mathrm{H}^{e}(f \star(g+h))(y) \sin (x y)\right) d y \\
& \quad=\frac{1}{\pi} \int_{0}^{\infty}\left(\mathrm{H}^{o}(f \star g+f \star h)(y) \cos (x y)+\mathrm{H}^{e}(f \star g+f \star h)(y) \sin (x y)\right) d y .
\end{aligned}
$$

Hence, the properties of HT odd and even parts, $\mathrm{H}^{o}, \mathrm{H}^{e}$, and that of the integral operator $\int$ imply that $\mathrm{HH}(f \star(g+h))(x)=\mathrm{HH}(f \star g+f \star h)(x)$.

Proof of (iv) is analogous to that given for part (ii).

This completes the proof.

Next is a straightforward corollary of Theorem 2.2.

Corollary 2.3 Let $f, g$ and $h$ be $L^{1}$ functions, then:

(i) $f \star g=g \star f$.

(ii) $(f \star g) \star h=f \star(g \star h)$.

(iii) $f \star(g+h)=f \star g+f \star h$.

(iv) $f+(g \star h)=(f+g) \star(f \star h)$. 


\section{$2.3 \mathrm{HT}$ and HHT of distributions}

In this subsection we discuss HT and HHT on a tempered distribution space.

Theorem 2.4 Iff is in $\mathcal{S}$, then $\mathrm{H} f$ is also in $\mathcal{S}$.

Proof If $f \in \mathcal{S}$, then HT certainly exists. Differentiating, in the ordinary sense, the righthand side of the integral equation

$$
(\mathrm{H} f)(x)=\int_{-\infty}^{\infty} f(\xi)(\cos (x \xi)+\sin (x \xi)) d \xi
$$

with respect to $x$ yields

$$
\frac{d}{d x}(\mathrm{H} f)(x)=\int_{-\infty}^{\infty} \xi f(\xi)(\cos (x \xi)-\sin (x \xi)) d \xi
$$

This is because the right-hand side of (9) converges uniformly for each $x$.

Indeed, integrating by parts $m$ times and by the fact that

$$
\frac{d^{m}}{d^{m} \xi}\left(\xi^{k} f(\xi)\right) \rightarrow 0
$$

as $\xi \rightarrow \infty$ for each $m \in \mathbb{N} \cup\{0\}$, we get

$$
\left|x^{m} \frac{d^{k}}{d^{k} x}(\mathrm{H} f)(x)\right| \leq \int_{-\infty}^{\infty}\left|\frac{d^{m}}{d^{m \xi}}\left(\xi^{k} f(\xi)\right)\right| d \xi .
$$

Since $f \in \mathcal{S}$, the integral on the right-hand side of (10) is bounded by constants, say $A_{m k}$. Hence

$$
\left|x^{m} \frac{d^{k}}{d^{k} x}(\mathrm{H} f)(x)\right| \leq A_{m k}, \quad-\infty<x<\infty
$$

for every pair of non-negative $m$ and $k$. This completes the proof.

Corollary 2.5 If $f$ is in $\mathcal{S}$, then $\mathrm{H}^{o} f$ and $\mathrm{H}^{e} f$ are in $\mathcal{S}$.

Corollary 2.6 Iff is in $\mathcal{S}$, then $\mathrm{HH} f$ is also in $\mathcal{S}$.

Proof By Corollary 2.5, $\mathrm{H}^{o} f, \mathrm{H}^{e} f \in \mathcal{S}$. Thus, the linearity of $\mathcal{S}$ and the fact that $\int \phi(\xi) d \xi \in$ $\mathcal{S}$ for every $\phi \in \mathcal{S}$ imply $\operatorname{HH} f \in \mathcal{S}$.

Let $f \in \mathcal{S}^{\prime}$, then, by the aid of Corollary 2.5 and Corollary 2.6, we are led to the following definitions:

$$
\langle\mathrm{H} f(x), \varphi(x)\rangle=\langle f(x), \mathrm{H} \varphi(x)\rangle
$$

and

$$
\langle\mathrm{HH} f(x), \varphi(x)\rangle=\langle f(x), \mathrm{HH} \varphi(x)\rangle .
$$


The right-hand sides of (11) and (12) are well defined and, therefore, from the left-hand sides of (11) and (12), we get that

$$
\mathrm{H} f(x), \mathrm{HH} f(x) \in \mathcal{S}^{\prime}
$$

for each $f \in \mathcal{S}^{\prime}$.

This can be stated in similar words as: HT and HHT of tempered distributions are tempered distributions.

Corollary 2.7 If $\phi \in \mathcal{S}$, then $(\mathrm{H} \phi)(\mathrm{HH} \phi) \in \mathcal{S}$.

Theorem 2.8 Let $f \in \mathcal{S}^{\prime}$, then $\mathrm{H} f$ and $\mathrm{HH} f$ are both linear mappings from $\mathcal{S}^{\prime}$ into $\mathcal{S}^{\prime}$.

Proof Let $f, g \in \mathcal{S}^{\prime}$ and $\varphi \in \mathcal{S}, \alpha \in \mathrm{R}$ be arbitrary, then

$$
\begin{aligned}
\langle\alpha \mathrm{H}(f+g)(x), \varphi(x)\rangle & =\langle\alpha(f+g)(x), \mathrm{H} \varphi(x)\rangle \\
& =\alpha\langle f(x), \mathrm{H} \varphi(x)\rangle+\alpha\langle g(x), \mathrm{H} \varphi(x)\rangle \\
& =\alpha\langle\mathrm{H} f(x), \varphi(x)\rangle+\alpha\langle\mathrm{H} g(x), \varphi(x)\rangle .
\end{aligned}
$$

Similarly, we proceed for $\mathrm{HH} f, \forall f \in \mathcal{S}^{\prime}$. Hence the theorem.

\section{Generalized distributions}

One of the youngest generalizations of functions, and more particularly of distributions, is the theory of Boehmians. The name Boehmian space is given to all objects defined by an abstract construction similar to that of field of quotients. The construction applied to function spaces yields various spaces of generalized functions.

For a linear space $\mathrm{Y}$ and a subspace $\mathrm{X}$ of $\mathrm{Y}$, assume that to all pairs $(f, \phi),(g, \psi)$ of elements, $f, g \in \mathrm{Y}, \phi, \psi \in \mathrm{X}$, the products $f * \phi, g * \psi$ are assigned such that the following conditions are satisfied:

(1) $\phi * \psi \in \mathrm{X}$ and $\phi * \psi=\psi * \phi$.

(2) $(f * \phi) * \psi=f *(\phi * \psi)$.

(3) $(f+g) * \phi=f * \phi+g * \phi$

(4) $k(f * \phi)=(k f) * \phi=f *(k \phi), k \in \mathrm{R}$.

Let $\Delta$ be a family of sequences from $\mathrm{X}$ such that for $f, g \in \mathrm{Y}$ then:

(5) If $\left(\epsilon_{n}\right) \in \Delta$ and $f * \epsilon_{n}=g * \epsilon_{n}, n=1,2, \ldots$, then $f=g$.

(6) $\left(\epsilon_{n}\right),\left(\tau_{n}\right) \in \Delta \Rightarrow\left(\epsilon_{n} * \tau_{n}\right) \in \Delta$.

The elements of $\Delta$ are called delta sequences.

Consider the class $\mathrm{A}$ of pairs of sequences defined by

$$
\mathrm{A}=\left\{\left(\left(f_{n}\right),\left(\epsilon_{n}\right)\right):\left(f_{n}\right) \subseteq \mathrm{Y}^{\mathrm{N}},\left(\epsilon_{n}\right) \in \Delta\right\}
$$

for each $n \in \mathrm{N}$.

The pair $\left(\left(f_{n}\right),\left(\epsilon_{n}\right)\right) \in \mathrm{A}$ is said to be quotient of sequences, denoted by $\frac{f_{n}}{\epsilon_{n}}$, if

$$
f_{n} * \epsilon_{m}=f_{m} * \epsilon_{n}, \quad \forall n, m \in \mathrm{N} .
$$


Two quotients of sequences $\frac{f_{n}}{\epsilon_{n}}$ and $\frac{g_{n}}{\tau_{n}}$ are said to be equivalent, $\frac{f_{n}}{\epsilon_{n}} \sim \frac{g_{n}}{\tau_{n}}$, if

$$
f_{n} * \epsilon_{m}=g_{m} * \tau_{n}, \quad \forall n, m \in \mathrm{N} .
$$

The relation $\sim$ is an equivalent relation on $\mathrm{A}$ and hence splits $\mathrm{A}$ into equivalence classes. The equivalence class containing $\frac{f_{n}}{\epsilon_{n}}$ is denoted by $\left[\frac{f_{n}}{\epsilon_{n}}\right]$. These equivalence classes are called Boehmians and the space of all Boehmians is denoted by $\mathrm{B}(\mathrm{Y}, \mathrm{X}, \Delta, *)$, see [8].

The sum and multiplication by a scalar of two Boehmians can be defined in a natural way

$$
\left[\frac{f_{n}}{\epsilon_{n}}\right]+\left[\frac{g_{n}}{\tau_{n}}\right]=\left[\frac{f_{n} * \tau_{n}+g_{n} * \epsilon_{n}}{\epsilon_{n} * \tau_{n}}\right]
$$

and

$$
a\left[\frac{f_{n}}{\epsilon_{n}}\right]=\left[\frac{a f_{n}}{\epsilon_{n}}\right], \quad a \text { being a complex number. }
$$

The operation $*$ and differentiation are defined by $\left[\frac{f_{n}}{\epsilon_{n}}\right] *\left[\frac{g_{n}}{\tau_{n}}\right]=\left[\frac{f_{n} * g_{n}}{\epsilon_{n} * \tau_{n}}\right]$ and $\mathcal{D}^{\alpha}\left[\frac{f_{n}}{\epsilon_{n}}\right]=\left[\frac{\mathcal{D}^{\alpha} f_{n}}{\epsilon_{n}}\right]$. Many times, $Y$ is equipped with the notion of convergence. The intrinsic relationship between the notion of convergence and the product $*$ are given by:

(1) If $f_{n} \rightarrow f$ as $n \rightarrow \infty$ in Y and, $\phi \in \mathrm{X}$ is any fixed element, then

$$
f_{n} * \phi \rightarrow f * \phi \quad \text { in Y as } n \rightarrow \infty
$$

(2) If $f_{n} \rightarrow f$ as $n \rightarrow \infty$ in Y and $\left(\epsilon_{n}\right) \in \Delta$, then $f_{n} * \epsilon_{n} \rightarrow f$ in Y as $n \rightarrow \infty$.

The operation $*$ is extended to $\mathrm{B}(\mathrm{Y}, \mathrm{X}, \Delta, *) \times \mathrm{X}$ by the following definition.

Definition 3.1 If $\left[\frac{f_{n}}{\epsilon_{n}}\right] \in \mathrm{B}(\mathrm{Y}, \mathrm{X}, \Delta, *)$ and $\phi \in \mathrm{X}$, then $\left[\frac{f_{n}}{\epsilon_{n}}\right] * \phi=\left[\frac{f_{n} * \phi}{\epsilon_{n}}\right]$.

In $\mathrm{B}(\mathrm{Y}, \mathrm{X}, \Delta, *)$, two types of convergence, $\delta$ and $\Delta$ convergence, are defined as follows.

Definition 3.2 A sequence of Boehmians $\left(\beta_{n}\right)$ in $\mathrm{B}(\mathrm{Y}, \mathrm{X}, \Delta, *)$ is said to be $\delta$-convergent to a Boehmian $\beta$ in $\mathrm{B}(\mathrm{Y}, \mathrm{X}, \Delta, *)$, denoted by $\beta_{n} \stackrel{\delta}{\rightarrow} \beta$, if there exists a delta sequence $\left(\epsilon_{n}\right)$ such that $\left(\beta_{n} * \epsilon_{n}\right),\left(\beta * \epsilon_{n}\right) \in \mathrm{Y}, \forall k, n \in \mathrm{N}$, and

$$
\left(\beta_{n} * \epsilon_{k}\right) \rightarrow\left(\beta * \epsilon_{k}\right) \quad \text { as } n \rightarrow \infty \text {, in Y, for every } k \in \mathrm{N} \text {. }
$$

The following lemma is equivalent to the statement of $\delta$-convergence.

Lemma 3.3 $\beta_{n} \stackrel{\delta}{\rightarrow} \beta(n \rightarrow \infty)$ in $\mathrm{B}(\mathrm{Y}, \mathrm{X}, \Delta, *)$ if and only if there are $f_{n, k}, f_{k} \in \mathrm{Y}$ and $\epsilon_{k} \in \Delta$ such that $\beta_{n}=\left[\frac{f_{n, k}}{\epsilon_{k}}\right], \beta=\left[\frac{f_{k}}{\epsilon_{k}}\right]$ and for each $k \in \mathrm{N}$,

$$
f_{n, k} \rightarrow f_{k} \quad \text { as } n \rightarrow \infty \text { in } \mathrm{Y}
$$

Definition 3.4 A sequence of Boehmians $\left(\beta_{n}\right)$ in $\mathrm{B}(\mathrm{Y}, \mathrm{X}, \Delta, *)$ is said to be $\Delta$-convergent to a Boehmian $\beta$ in $\mathrm{B}(\mathrm{Y}, \mathrm{X}, \Delta, *)$, denoted by $\beta_{n} \stackrel{\Delta}{\rightarrow} \beta$, if there exists an $\left(\epsilon_{n}\right) \in \Delta$ such that $\left(\beta_{n}-\beta\right) * \epsilon_{n} \in \mathrm{Y}, \forall n \in \mathrm{N}$, and $\left(\beta_{n}-\beta\right) * \epsilon_{n} \rightarrow 0$ as $n \rightarrow \infty$ in Y. See, for example, [7, 9-12] and $[2]$. 


\section{The spaces $\mathrm{B}_{1}\left(\mathcal{S}^{\prime}, \mathcal{S}, \Delta, *\right)$ and $\mathrm{B}_{2}\left(\Psi_{1}, \Psi_{2}, \Psi_{3}, \bullet\right)$}

Theorem 4.1 (Second convolution theorem of HHT) Let $f$ and $g$ be $\mathrm{L}^{1}$ functions, then

$$
\mathrm{HH}(f * g)(x)=2 \mathrm{HH}\left(\mathrm{H}^{-1}(\mathrm{H} f \mathrm{H} g)\right)(x),
$$

where $*$ is the usual convolution product of $f$ and $g$, see [1].

Proof Using the definition of HHT implies

$$
\mathrm{HH}(f * g)(x)=\int_{0}^{\infty}(\mathrm{A}(\xi) \cos (x \xi)+\mathrm{B}(\xi) \sin (x \xi)) d \xi
$$

with $\mathrm{A}(\xi)=\mathrm{H}^{o}(f * g)(\xi)$ and $\mathrm{B}(\xi)=\mathrm{H}^{e}(f * g)(\xi)$.

Fubini's theorem implies

$$
\mathrm{A}(\xi)=\int_{-\infty}^{\infty} f(z) \int_{-\infty}^{\infty} g(t-z) \sin (t \xi) d t d z .
$$

The substitution $t-z=y$ and the fact

$$
\sin (y+z) \xi=\sin (y \xi) \cos (z \xi)+\cos (y \xi) \sin (z \xi)
$$

imply

$$
\mathrm{A}(\xi)=\mathrm{H}^{e} f(\xi) \mathrm{H}^{o} f(\xi)+\mathrm{H}^{o} f(\xi) \mathrm{H}^{e} f(\xi) .
$$

Invoking

$$
\begin{aligned}
\mathrm{H}^{e} f(\xi)=\frac{\mathrm{H} f(\xi)+\mathrm{H} f(-\xi)}{2}, & \mathrm{H}^{o} f(\xi)=\frac{\mathrm{H} f(\xi)-\mathrm{H} f(-\xi)}{2}, \\
\mathrm{H}^{e} g(\xi)=\frac{\mathrm{H} g(\xi)+\mathrm{H} g(-\xi)}{2}, & \mathrm{H}^{o} g(\xi)=\frac{\mathrm{H} g(\xi)-\mathrm{H} g(-\xi)}{2}
\end{aligned}
$$

in (14) then multiplying and canceling similar quantities yield

$$
\mathrm{A}(\xi)=(\mathrm{H} f \mathrm{H} g)(\xi)+(\mathrm{H} f \mathrm{H} g)(-\xi)=\mathrm{H}\left(\mathrm{H}^{-1}(\mathrm{H} f \mathrm{H} g)\right)(z)+\mathrm{H}\left(\mathrm{H}^{-1}(\mathrm{H} f \mathrm{H} g)\right)(-z)
$$

or

$$
\mathrm{A}(\xi)=2 \mathrm{H}^{e}\left(\mathrm{H}^{-1}(\mathrm{H} f \mathrm{Hg})\right)(\xi) .
$$

Similarly, we proceed for $\mathrm{B}(\xi)$ to get

$$
\mathrm{B}(\xi)=2 \mathrm{H}^{o}\left(\mathrm{H}^{-1}(\mathrm{H} f \mathrm{Hg})\right)(\xi) .
$$

Hence, invoking (16) and (17) in (13), our theorem follows. 
Denote by $\mathrm{B}_{1}\left(\mathcal{S}^{\prime}, \mathcal{S}, \Delta, *\right)$ the usual Boehmian space with the convolution product $*$ as an operation, $\mathcal{S}^{\prime}$ as a group, $\mathcal{S}$ as a subgroup of $\mathcal{S}^{\prime}\left(\mathcal{S}\right.$ dense in $\mathcal{S}^{\prime}$ ) and, $\Delta$ as the collection of delta sequences from $\mathcal{S}$ such that

$$
\begin{aligned}
& \Delta_{1} \int \delta_{n}(x) d x=1, \\
& \Delta_{2} \int\left|\delta_{n}(x)\right| d x<\mathrm{M}, \quad 0<\mathrm{M} \in \mathrm{R}, \\
& \Delta_{3} \operatorname{supp} \delta_{n}(x) \rightarrow 0 \quad \text { as } n \rightarrow \infty .
\end{aligned}
$$

Let us consider another space of Boehmians:

Denote by $\Psi_{1}$ the space of HHTs of distributions from $\mathcal{S}^{\prime}$. Indeed, $\Psi_{1}$ is also a subspace of $\mathcal{S}^{\prime}$ by (12). A member $\xi_{n} \in \Psi_{1}$ is said to converge in $\Psi_{1}$ to a value $\xi$ if there are $\tau_{n}, \tau \in \mathcal{S}^{\prime}$ such that $\tau_{n}$ reaches $\tau$ for large values of $n$.

Also, denote by $\Psi_{2}$ the set of HHTs of test functions from $\mathcal{S}$, then $\Psi_{2}$ is a subspace of $\Psi_{1}$ by Corollary 2.6. In similar notations $\Psi_{3}=\mathrm{HH} \Delta$.

Next, let us consider an operation $\bullet: \Psi_{1} \times \Psi_{2} \rightarrow \Psi_{1}$ defined by

$$
\bullet(\xi, \phi)(x)=2 \mathrm{HH}\left(\mathrm{H}^{-1}\left(\mathrm{H}^{*} \mathrm{H}^{*}\right)\right)(x)
$$

for $\xi=H H \xi^{*}, \phi=H H \phi^{*}$

Theorem 4.2 Let $\xi \in \Psi_{1}$ and $\phi \in \Psi_{2}$, then for $\xi=H H \xi^{*}$ and $\phi=H H \phi^{*}$,

$$
\bullet(\xi, \phi)=\mathrm{HH}\left(\xi^{*} * \phi^{*}\right)
$$

Proof For every $\xi \in \Psi_{1}, \phi \in \Psi_{2}$, we get

$$
\begin{aligned}
\bullet(\xi, \phi)(x) & =2 \mathrm{HH}\left(\mathrm{H}^{-1}\left(\mathrm{H}^{*} \mathrm{H} \phi^{*}\right)\right)(x) \\
& =\mathrm{HH}\left(\xi^{*} * \phi^{*}\right)(x),
\end{aligned}
$$

where $\xi=\mathrm{HH} \xi^{*}, \phi=\mathrm{HH} \phi^{*}$. This proves the theorem.

Theorem 4.3 Let $\phi_{1}, \phi_{2} \in \Psi_{2}$, then $\bullet\left(\phi_{1}, \phi_{2}\right)=\bullet\left(\phi_{1}, \phi_{2}\right)$.

Proof Using (17) we get

$$
\bullet\left(\phi_{1}, \phi_{2}\right)(x)=2 \mathrm{HH}\left(\mathrm{H}^{-1}\left(\mathrm{H} \phi_{1}^{*} \mathrm{H} \phi_{2}^{*}\right)\right)(x) \text {, }
$$

where $\phi_{1}=H H \phi_{1}^{*}, \phi_{2}=H H \phi_{2}^{*}$.

By (18) and Theorem 4.2, we get

$$
\begin{aligned}
\bullet\left(\phi_{1}, \phi_{2}\right)(x) & =\mathrm{HH}\left(\phi_{1}^{*} * \phi_{2}^{*}\right)(x) \\
& =\mathrm{HH}\left(\phi_{2}^{*} * \phi_{1}^{*}\right)(x) \\
& =2 \mathrm{HH}\left(\mathrm{H}^{-1}\left(\mathrm{H} \phi_{2}^{*} \mathrm{H} \phi_{1}^{*}\right)\right)(x)=\bullet\left(\phi_{2}, \phi_{1}\right)(x) .
\end{aligned}
$$

Hence the theorem. 
Theorem 4.4 Let $\xi_{1}, \xi_{2}, \xi_{n}, \xi \in \Psi_{1}$ and $\phi \in \Psi_{2}$, then:

(i) $\bullet\left(k \xi_{1}, \phi\right)(x)=\bullet\left(\xi_{1}, k \phi\right)(x)=k\left(\bullet\left(\xi_{1}, \phi\right)(x)\right), k \in \mathrm{R}$.

(ii) $\bullet\left(\xi_{1}+\xi_{2}, \phi\right)(x)=\bullet\left(\xi_{1}, \phi\right)(x)+\bullet\left(\xi_{2}, \phi\right)(x)$.

(iii) $\bullet\left(\xi_{n}, \phi\right)(x) \rightarrow \bullet(\xi, \phi)(x)$ as $n \rightarrow \infty$.

Proof (i) The linearity of HHTs and (17) implies

$$
\begin{aligned}
\bullet(k \xi, \phi)(x) & =2 \mathrm{HH}\left(\mathrm{H}^{-1}\left(k \mathrm{H} \xi^{*} \mathrm{H} \phi^{*}\right)\right)(x) \\
& =2 \mathrm{HH}\left(\mathrm{H}^{-1}\left(\mathrm{H} \xi^{*}\left(k \mathrm{H} \phi^{*}\right)\right)\right)(x) \\
& =2 \mathrm{HH}\left(\mathrm{H}^{-1}\left(\mathrm{H}^{*} \mathrm{H}\left(k \phi^{*}\right)\right)\right)(x), \quad \text { by the linearity of HT } \\
& =\bullet(\xi, k \phi)(x) .
\end{aligned}
$$

Similarly,

$$
\bullet(k \xi, \phi)(x)=k\left(\bullet\left(\xi_{1}, \phi\right)(x)\right) \text {. }
$$

The proof of (ii) and (iii) follows from simple computations. The proof is therefore completed.

Theorem 4.5 Let $\left(\alpha_{n}\right),\left(\varepsilon_{n}\right) \in \Psi_{3}$, then $\bullet\left(\alpha_{n}, \varepsilon_{n}\right) \in \Psi_{3}$.

Proof For $\left(\alpha_{n}\right),\left(\varepsilon_{n}\right) \in \Psi_{3}$,

$$
\begin{aligned}
\text { - }\left(\alpha_{n}, \varepsilon_{n}\right)(x) & =2 \mathrm{HH}\left(\mathrm{H}^{-1}\left(\mathrm{H} \alpha_{n}^{*} \mathrm{H} \varepsilon_{n}^{*}\right)\right)(x) \\
& =\mathrm{HH}\left(\alpha_{n}^{*} * \varepsilon_{n}^{*}\right)(x) .
\end{aligned}
$$

Since $\alpha_{n}^{*} * \varepsilon_{n}^{*} \in \Delta$, we get

$$
\bullet\left(\alpha_{n}, \varepsilon_{n}\right)(x) \in \mathrm{HH} \Delta
$$

The proof is completed.

Theorem 4.6 Let $\xi \in \Psi_{1}, \phi_{1}, \phi_{2} \in \Psi_{2}$, then

$$
\bullet\left(\bullet\left(\xi, \phi_{1}\right), \phi_{2}\right)(x)=\bullet\left(\xi, \bullet\left(\phi_{1}, \phi_{2}\right)\right)(x) .
$$

Proof Follows from similar computations to those above.

In detail, for $\phi_{1}=H H \phi_{1}^{*}, \phi_{2}=H H \phi_{2}^{*}$ and $\xi=H H \xi^{*}$, we see that

$$
\begin{aligned}
\bullet\left(\bullet\left(\xi, \phi_{1}\right), \phi_{2}\right)(x) & =\mathrm{HH}\left(\bullet\left(\xi, \phi_{1}\right)^{*} * \phi_{2}^{*}\right)(x) \\
& =\mathrm{HH}\left(\left(\mathrm{HH}\left(\xi^{*} * \phi_{1}^{*}\right)\right)^{*} * \phi_{2}^{*}\right)(x) \\
& =\mathrm{HH}\left(\left(\xi^{*} * \phi_{1}^{*}\right) * \phi_{2}^{*}\right)(x) \\
& =\mathrm{HH}\left(\xi^{*} *\left(\phi_{1}^{*} * \phi_{2}^{*}\right)\right)(x)
\end{aligned}
$$




$$
\begin{aligned}
& =\mathrm{HH}\left(\xi^{*} *\left(\phi_{1}^{*} * \phi_{2}^{*}\right)\right)(x) \\
& =\bullet\left(\bullet \xi,\left(\phi_{1}, \phi_{2}\right)\right)(x)
\end{aligned}
$$

Hence our theorem is completely proved.

Theorem 4.7 Let $\xi_{1}, \xi_{2} \in \Psi_{1}$ and $\left(\delta_{n}\right) \in \Psi_{3}$ and $\bullet\left(\xi_{1}, \delta_{n}\right)(x)=\bullet\left(\xi_{2}, \delta_{n}\right)(x)$, then $\xi_{1}=\xi_{2}$.

Proof Assume that $\bullet\left(\xi_{1}, \delta_{n}\right)(x)=\bullet\left(\xi_{2}, \delta_{n}\right)(x)$, then

$$
2 \mathrm{HH}\left(\mathrm{H}^{-1}\left(\mathrm{H} \xi_{1}^{*} \mathrm{H} \delta_{n}^{*}\right)\right)(x)=2 \mathrm{HH}\left(\mathrm{H} \xi_{2}^{*} \mathrm{H} \delta_{n}^{*}\right)(x) .
$$

Hence, $\mathrm{HH}\left(\xi_{1}^{*} * \delta_{n}^{*}\right)(x)=\mathrm{HH}\left(\xi_{2}^{*} * \delta_{n}^{*}\right)(x)$. Allowing $n \rightarrow \infty$ implies

$$
\mathrm{HH}\left(\xi_{1}^{*}\right)=\mathrm{HH}\left(\xi_{2}^{*}\right)
$$

Hence $\xi_{1}=\xi_{2}$. This completes the proof.

Theorem 4.8 Let $\left(\delta_{n}\right) \in \Psi_{3}$ and $\xi \in \Psi_{1}$, then

$$
\bullet\left(\xi, \delta_{n}\right) \rightarrow \xi \quad \text { as } n \rightarrow \infty
$$

Proof Since $\xi \in \Psi_{1},\left(\delta_{n}\right) \in \Psi_{3}$, there are $\xi^{*} \in \mathcal{S}, \delta_{n}^{*} \in \Delta$ such that $\mathrm{HH} \xi^{*}=\xi$ and $\delta_{n}=\mathrm{HH} \delta_{n}^{*}$. Hence

$$
\begin{aligned}
\bullet\left(\xi, \delta_{n}\right)(x) & =2 \mathrm{HH}\left(\mathrm{H}^{-1}\left(\mathrm{H} \xi^{*} \mathrm{H} \delta_{n}^{*}\right)\right)(x) \\
& =\mathrm{HH}\left(\xi^{*} * \delta_{n}^{*}\right)(x) \rightarrow \mathrm{HH} \xi^{*}=\xi
\end{aligned}
$$

as $n \rightarrow \infty$ since $\delta_{n}^{*} \in \Delta$.

Thus the theorem.

The Boehmian space $\mathrm{B}_{2}\left(\Psi_{1}, \Psi_{2}, \Psi_{3}, \bullet\right)$ is completely established.

A typical element in $\mathrm{B}_{2}\left(\Psi_{1}, \Psi_{2}, \Psi_{3}, \bullet\right)$ is given as

$$
\left[\frac{\mathrm{HH} f_{n}}{\mathrm{HH} \phi_{n}}\right] .
$$

The concept of quotients of sequences is justified by

$$
\begin{aligned}
\bullet\left(\mathrm{HH} f_{n}, \mathrm{HH} \phi_{m}\right) & =2 \mathrm{HH}\left(\mathrm{H}^{-1}\left(\mathrm{H} f_{n} \mathrm{H} \phi_{m}\right)\right) \\
& =\mathrm{HH}\left(f_{n} * \phi_{m}\right) \\
& =\mathrm{HH}\left(f_{m} * \phi_{n}\right) \\
& =\mathrm{HH}\left(\mathrm{H}^{-1}\left(\mathrm{H} f_{m} \mathrm{H} \phi_{n}\right)\right) \\
& =\bullet\left(\mathrm{HH} f_{m}, \mathrm{HH} \phi_{n}\right) .
\end{aligned}
$$

Hence, $\bullet\left(\mathrm{HH} f_{n}, \mathrm{HH} \phi_{m}\right)=\bullet\left(\mathrm{HH} f_{m}, \mathrm{HH} \phi_{n}\right)$. 
Two quotients $\frac{\mathrm{HH} f_{n}}{\mathrm{HH} \phi_{n}}$ and $\frac{\mathrm{HH} g_{n}}{\mathrm{HH} \tau_{n}}$ are said to be equivalent in the sense of $\mathrm{B}_{2}\left(\Psi_{1}, \Psi_{2}, \Psi_{3}, \bullet\right)$ if

$$
\bullet\left(\mathrm{HH} f_{n}, \mathrm{HH} \tau_{m}\right)=\bullet\left(H H g_{m}, \mathrm{HH} \phi_{n}\right)
$$

Sum and multiplication by a scalar of two Boehmians can be defined in a natural way

$$
\left[\frac{\mathrm{HH} f_{n}}{\mathrm{HH} \phi_{n}}\right]+\left[\frac{\mathrm{HH} g_{n}}{\mathrm{HH} \tau_{n}}\right]=\left[\frac{\mathrm{HH} f_{n} \bullet \mathrm{HH} \tau_{n}+\mathrm{HH} g_{n} \bullet \mathrm{HH} \phi_{n}}{\mathrm{HH} \phi_{n} \bullet \mathrm{HH} \tau_{n}}\right]
$$

and

$$
\mathcal{A}\left[\frac{\mathrm{HH} f_{n}}{\mathrm{HH} \phi_{n}}\right]=\left[\frac{\mathcal{A H H} f_{n}}{\mathrm{HH} \phi_{n}}\right], \quad \mathcal{A} \text { being a complex number. }
$$

The operation $\bullet$ and differentiation are defined by

$$
\left[\frac{\mathrm{HH} f_{n}}{\mathrm{HH} \phi_{n}}\right] \bullet\left[\frac{\mathrm{HH} g_{n}}{\mathrm{HH} \tau_{n}}\right]=\left[\frac{\mathrm{HH} f_{n} \bullet \mathrm{HH} g_{n}}{\mathrm{HH} \phi_{n} \bullet \mathrm{HH} \tau_{n}}\right]
$$

and

$$
\mathcal{D}^{\alpha}\left[\frac{\mathrm{HH} f_{n}}{\mathrm{HH} \phi_{n}}\right]=\left[\frac{\mathcal{D}^{\alpha} \mathrm{HH} f_{n}}{\mathrm{HH} \phi_{n}}\right] \text {. }
$$

\section{HHT of Boehmians}

Let us define the EHHT of a Boehmian $\left[\frac{\mathrm{HH} f_{n}}{\mathrm{HH} \phi_{n}}\right] \in \mathrm{B}_{1}\left(\mathcal{S}^{\prime}, \mathcal{S}, \Delta, *\right)$ by

$$
\Lambda\left[\frac{\mathrm{HH} f_{n}}{\mathrm{HH} \phi_{n}}\right]=\left[\frac{\mathrm{HH} f_{n}}{\mathrm{HH} \phi_{n}}\right] \in \mathrm{B}_{2}\left(\Psi_{1}, \Psi_{2}, \Psi_{3}, \bullet\right)
$$

It is clear that EHHT is well defined.

Theorem 5.1 EHHT is linear.

Theorem 5.2 EHHT is one-to-one.

Theorem 5.3 EHHT is continuous with respect to $\delta$ convergence.

Proof Let $\beta_{n} \stackrel{\delta}{\rightarrow} \beta$ in $\mathrm{B}_{1}\left(\mathcal{S}^{\prime}, \mathcal{S}, \Delta, *\right)$ as $n \rightarrow \infty$. We show that $\Lambda \beta_{n} \rightarrow \Lambda \beta$ in $\mathrm{B}_{2}\left(\Psi_{1}, \Psi_{2}\right.$, $\left.\Psi_{3}, \bullet\right)$ as $n \rightarrow \infty$.

For each $\beta_{n}, \beta \in \mathrm{B}_{1}\left(\mathcal{S}^{\prime}, \mathcal{S}, \Delta, *\right)$ we, by [12], can find $f_{n, k}, f_{k} \in \mathcal{S}^{\prime}$ such that

$$
\beta_{n}=\left[\frac{f_{n, k}}{\phi_{k}}\right]
$$

and $\beta=\left[\frac{f_{k}}{\phi_{k}}\right]$ and $f_{n, k} \rightarrow f_{k}$ as $n \rightarrow \infty, \forall k \in \mathrm{N}$.

The continuity of HHTs implies

$$
\mathrm{HH} f_{n, k} \rightarrow \mathrm{HH} f_{k} \quad \text { as } n \rightarrow \infty \text { in } \Psi_{1},
$$


and hence

$$
\frac{\mathrm{HH} f_{n, k}}{\mathrm{HH} \phi_{k}} \sim \frac{\mathrm{HH} f_{k}}{\mathrm{HH} \phi_{k}} .
$$

Thus,

$$
\beta_{n}=\left[\frac{\mathrm{HH} f_{n, k}}{\mathrm{HH} \phi_{k}}\right] \rightarrow \beta\left[\frac{\mathrm{HH} f_{k}}{\mathrm{HH} \phi_{k}}\right] \text { as } n \rightarrow \infty \text { in } \beta_{2}\left(\Psi_{1}, \Psi_{2}, \Psi_{3}, \bullet\right) .
$$

The proof is completed.

Theorem 5.4 EHHTs are continuous with respect to $\Delta$ convergence.

Proof Let $\beta_{n} \stackrel{\Delta}{\rightarrow} \beta$ in $\mathrm{B}_{1}\left(\mathcal{S}^{\prime}, \mathcal{S}, \Delta, *\right)$ as $n \rightarrow \infty$, then there are $f_{n} \in \mathcal{S}^{\prime}$ and $\phi_{n} \in \Delta$ such that

$$
\left(\beta_{n}-\beta\right) * \phi_{n}=\left[\frac{f_{n} * \phi_{k}}{\phi_{k}}\right]
$$

and $f_{n} \rightarrow 0$ as $n \rightarrow \infty$. Hence, by Theorem 4.1,

$$
\begin{aligned}
\mathrm{HH}\left(\left(\beta_{n}-\beta\right) * \phi_{n}\right) & =\mathrm{HH}\left[\frac{f_{n} * \phi_{k}}{\phi_{k}}\right] \\
& =\left[\frac{\mathrm{HH}\left(f_{n} * \phi_{k}\right)}{\mathrm{HH} \phi_{k}}\right] \simeq \mathrm{HH} f_{n} \rightarrow \infty \quad \text { as } n \rightarrow \infty .
\end{aligned}
$$

Hence the theorem.

Remark 5.5 Let $\beta=\left[\frac{\mathrm{HH} f_{n}}{\mathrm{HH} \delta_{n}}\right] \in \mathrm{B}_{2}\left(\Psi_{1}, \Psi_{2}, \Psi_{3}, \bullet\right)$, then it is so natural to define the inverse HHT of $\beta$ as

$$
\Lambda^{-1} \beta=\left[\frac{f_{n}}{\delta_{n}}\right]
$$

in the space $\mathrm{B}_{1}\left(\mathcal{S}^{\prime}, \mathcal{S}, \Delta, *\right)$.

It is of interest to see that the inverse transform $\Lambda^{-1}$ preserves all the above properties that $\Lambda$ does such as linearity, one-to-one and continuity of $\Lambda^{-1}$ with respect to convergence in $\mathrm{B}_{1}\left(\mathcal{S}^{\prime}, \mathcal{S}, \Delta, *\right)$. Proofs are avoided.

Competing interests

The authors declare that they have no competing interests.

Authors' contributions

Both authors contributed equally to the manuscript and read and approved the final draft.

\section{Author details}

'Department of Applied Sciences, Faculty of Engineering Technology, Al-Balqa Applied University, Amman, 11134, Jordan. ${ }^{2}$ Department of Mathematics and Institute of Mathematical Research, Universiti Putra Malaysia (UPM), Serdang, Selangor 43400, Malaysia.

\section{Acknowledgements}

The authors are very grateful to the referees for their valuable suggestions and comments that helped to improve the paper 


\section{References}

1. Pathak, RS: Integral Transforms of Generalized Functions and Their Applications. Gordon \& Breach, New York (1997)

2. Zemanian, AH: Generalized Integral Transformation. Dover, New York (1987)

3. Hartley, RVL: A more symmetrical Fourier analysis applied to transmission problems. Proc. IRE 30(2), 144-150 (1942)

4. Millane, RP: Analytic properties of the Hartley transform and their applications. Proc. IEEE 82(3), 413-428 (1994)

5. Al-Omari, SKQ, Kılıçman, A: Note on Boehmians for class of optical Fresnel wavelet transforms. J. Funct. Spaces Appl. 2012, Article ID 405368 (2012). doi:10.1155/2012/405368

6. Sundarajan, N: Fourier and Hartley transforms: a mathematical twin. Indian J. Pure Appl. Math. 8(10), 1361-1365 (1997)

7. Al-Omari, SKQ, Kılıçman, A: On generalized Hartley-Hilbert and Fourier-Hilbert transforms. Adv. Differ. Equ. 2012, 232 (2012). doi:10.1186/1687-1847-2012-232

8. Al-Omari, SKQ, Kılıçman, A: On diffraction Fresnel transforms for Boehmians. Abstr. Appl. Anal. 2011, Article ID 712746 (2011) doi:10.1155/2011/712746

9. Boehme, TK: The support of Mikusinski operators. Trans. Am. Math. Soc. 176, 319-334 (1973)

10. Mikusinski, P: Fourier transform for integrable Boehmians. Rocky Mt. J. Math. 17(3), 577-582 (1987)

11. Mikusinski, P: Tempered Boehmians and ultradistributions. Proc. Am. Math. Soc. 123(3), 813-817 (1995)

12. Sundararajan, N, Srinivas, Y: Fourier-Hilbert versus Hartley-Hilbert transforms with some geophysical applications J. Appl. Geophys. 71, 157-161 (2010)

doi:10.1186/1687-1847-2013-222

Cite this article as: Al-Omari and Kllıçman: On the generalized Hartley and Hartley-Hilbert transformations. Advances in Difference Equations 2013 2013:222.

\section{Submit your manuscript to a SpringerOpen ${ }^{\circ}$ journal and benefit from:}

- Convenient online submission

- Rigorous peer review

- Immediate publication on acceptance

- Open access: articles freely available online

- High visibility within the field

- Retaining the copyright to your article 